\title{
The Relationship between Cognitive Processing and Reading
}

\author{
Ooi Boon Keat \& Khaidzir bin Hj. Ismail \\ Psychology and Human Development Department \\ Faculty of Social Science and Humanities, Universiti Kebangsaan Malaysia, Malaysia \\ E-mail: gunehmia@yahoo.com, izay@ukm.my
}

Received: April 28, 2011

doi:10.5539/ass.v7n10p44
Accepted: June 17, $2011 \quad$ Published: October 1, 2011

URL: http://dx.doi.org/10.5539/ass.v7n10p44

\begin{abstract}
This study discusses the Cognitive Assessment System (CAS) in assessing cognitive processing. CAS is capable of measuring not only the cognitive ability and process but also of able to determine the deficits in relation with specific reading skills. Therefore, this study aims to examine the relationship of PASS cognitive processes with the reading components of Wide Range Achievement Test-4 (WRAT-4). The CAS and WRAT-4 were administered to a hundred primary standard three Malaysian children but based on American norms. The full scale of CAS mean scores was 98 which is the average level of cognitive processing among the subjects. While the WRAT-4 reading components showed the mean score of overall reading performance was 90 which slightly reach the average level. Correlation analysis revealed that cognitive processes were significantly correlated with overall reading performance with $r=0789, p<0.01$. Simultaneous and Planning processing had quite strong relationship with the overall reading performance which was $r=0.724, p<0.01$ and $r=0.676, p<0.01$ respectively. However, Successive processing had low score of $r=0.393, p<0.01$ for the correlation with reading comprehension. CAS is one of the alternatives in providing such capabilities in measuring cognitive processes that could predict reading deficits and relevant to identify performance on reading-related skills.
\end{abstract}

Keywords: Cognitive Assessment System (CAS), PASS cognitive processes, Reading, English as Second Language (ESL)

\section{INTRODUCTION}

Human cognition comprises of every activity that is involved in the thinking processes. It covers the basic theory of information processing, social cognitive perspective, the developmental cognition theory and also neuropsychological points of view. These theoretical explanations have given an overall thought that contributed to the understanding of PASS theory which was the focus of this present study. The combination of the cognitive processing and functions of brain served as the foundation explaining the reading processes that being involved in the study. The theoretical framework of this study explained learning to read in specific as the relationship between reading and the cognitive processing. With respect to the links between the cognitive development theories stated above, the principle of human cognition was applied to the points of view like the neuropsychological and the cognitive functioning and processing of learning a language. This study discussed either directly or indirectly the cognitive processing of PASS in relation with reading component skills. They are relevant to be discussed in order to get better understanding on the alternative of PASS cognitive processing theory as suggested by Naglieri et al (2006), CAS is a cognitive approach to reconceptualizing intelligence offers a viable alternative to a traditional general intelligence approach that $g$ factor. Naglieri et al. (2007) compared the performance of referred bilingual Hispanic children on the Planning, Attention, Simultaneous, Successive (PASS) theory as measured by English and Spanish versions of the Cognitive Assessment System (CAS; Naglieri \& Das 1997a). Small mean differences were noted between the means of the English and Spanish versions for the Simultaneous and Successive processing scales. The findings showed that these children performed consistently as cognitive processing despite the language difference.

Reading in English is unlike the writing system of many other languages, like Malay, Chinese and Hindu, the sounds associated with particular letters in English are not entirely predictable. Students who are learning English as a second language usually do so in an environment where other individuals predominantly speak English. What is unique to the current study is that students are learning English as a foreign language in an environment with predominantly non-English speaking individuals. The primary students who most of them are 
Malays and some Indians in the present research speak their mother tongue fluently. Their exposure to English reading and writing began when they entered kindergarten. What influence such a multilingual literacy and language environments might have on English reading and comprehension was examined by Mishra and Stainthorp (2007) in a longitudinal study beginning at kindergarten. In fact, the objective of that project was to determine cross-linguistic development in regard to reading. As the authors observed, learning to read English consistently requires more fine-grained phonological analysis at the level of phonemes than does learning to read Oriya. On the other hand, learning to speak, read, and writes Oriya equips children with the skills to analyze words at the level of syllables and whole words. Other research also has suggested that cross-language transfer exists for ESL readers (e.g. Lesaux, Lipka \& Siegel 2006).

Elleman (2009) explained that providing early intervention for children with poor comprehension is crucial on accurate remedial. There is a concern that the insensitivity of reading comprehension level may be impeding early identification and intervention of reading comprehension deficits. This is an important implication for CAS as an alternative and possibility to provide appropriate identification for the cognitive processing factor of reading comprehension. Dirks et al. (2008), children with combined reading and arithmetic disabilities seem to have more generalized achievement difficulties than single-deficit groups. Shapiro et al. (2008) reported the importance of speech processing skills and other baseline skills such as IQ and memory, motor, rhyme, speed and accuracy were correlated with the crucial predictive skills. These findings are relevant to discuss the successive process of PASS theory which involves speech and auditory processing as the basic required skills in reading and phoneme skills. Also, previous studies reported children who have difficulties in reading such as Dyslexia are having weakness in successive cognitive processing. Siegel \& Lipka (2007) found that English as a Second Language (ESL) children developed strong reading skills and their status as ESL speakers did not put them at risk for reading difficulties in Standard 3.

Das, Naglieri \& Kirby (1994) found that children with reading decoding failure and phonological coding problems perform poorly in successive processing and are associated with assembly of correct sounds in order which demands successive processing. On the other hand, student who scores high in planning is aware of the task-strategy relationship and monitors strategic success which are two essential aspects of metacognition. According to Naglieri (1999), PASS processes are being associated with phonological components of spoken and written language. Planning is said to be associated with the efficient execution and verification of speaking and reading words. Attention corresponds to the alertness to discrete sounds and letters, and inhibiting irrelevant stimuli. Successive processing is associated with sequentially decoding the sounds of words or making one to one correspondences with letters and sounds. Simultaneous processing is associated with surveying all the elements of a word and acquiring the sound and letter patterns in a rather hierarchical manner (i.e. understanding that certain letters cue the sounds of other letters in words - such as 'e' at the end of the word 'came' cues the reader to say the ' $a$ ' as a long vowel sound). According to Khaidzir \& Ooi (2008), the poor achievers of English as Second Language (ESL) children were significantly low for simultaneous processing which is highly related to the ability of comprehension.

Joseph, McCachran and Naglieri (2003) studied the relationship among cognitive processing, phonological processing and basic reading skill performance. The study also aimed to determine which PASS cognitive processes best predicted phonological processes, which were best predictors of basic word-recognition performance. The study involved 62 primary-grade children ( 40 males and 22 females) with ages range from seven years to nine years. The sample children are referred for reading problems and they were given measurement of cognitive process (CAS), phonological processes (comprehension Test of Phonological Processing) and basic reading achievement (Woodcock-Johnson Tests of Academic Achievement-III). In general, the subjects of study showed the same characteristics of lower successive processing score. This could be explained by the predictor of phonological memory which was strongly related to the successive processing. The study also indicated that there were significant relationships between simultaneous processing, letter-word identification and word attack. There were also significant relationships between planning and letter-word identification. Moreover, it also showed that the two cognitive processes (successive and simultaneous processes) are related to decoding process in reading which comprises phonological processing.

Besides the specific studies on the reading skills, PASS cognitive processing was also used in determining learning skills. Fein and Day (2004) studied the PASS theory of intelligence and the acquisition of a complex skill by examining the criterion-related validity of CAS scores with respect to the acquisition of a complex skill. It was acceptable to link the successive processing with overall performance of all learning for computer-based task. This is because the Successive process is the systematic process that require a person to process information in order which is the must process in aviation. The simultaneous processing provides the overall understanding of the links 
for every task to perform at the better effect of the task. While, attention is the process of cognition that requires focus, a selective and sustainable effort in completing a task and it was relevant to the skill-based learning measure. The non-significant score of Planning processing might be the reason of the dynamic aviation environment itself that need not much strategy and meta-cognitive processing compare to systematic and order processing for carrying out the tasks.

\section{PASS THEORY}

According to Luria's PASS theory, there are three types of cognitive processes responsible for mental activity associated with three functional units of the brain. These processes refer to the mental activities which involved attention (first unit), simultaneous and successive processing (second unit), and planning (third unit) cognitive processes. The first functional unit, located in the brain stem and reticular activating system, provides the brain with the appropriate level of arousal or cortical tone for focused attention and resistance to distraction. The second functional unit (occipital-parietal and frontal-temporal areas of the brain) is responsible for "receiving, analyzing and storing information" using simultaneous and successive processing. The third functional unit is located in the frontal lobes of the brain and is responsible for planning, including the programming, regulation, and verification of behavior (Luria 1973: 67). This provides the capability for behavior such as asking questions and problem solving and the capacity for self-monitoring (Das et al. 1994). These processes provide a different perspective that redefines intelligence within the context of cognitive processes (Naglieri 1999).

Planning is a cognitive process that involves selecting and using strategies in decision making and problem solving. This process is interrelated to other process of PASS. It acquires efficient solution methods and best strategies which can be used in planning the ways to solve problems. According to Naglieri and Das (1997c), "planning is a mental process by which the individual determines, selects, applies, and evaluates solutions to problems". This process requires the ways to solve problems of varying complexity and may involve attention, simultaneous, and successive processes as well as knowledge. According to Naglieri (1999) planning is central to all activities in which there are both intentionality and a need for some method to solve a problem. This process includes self-monitoring and impulse control as well as plan generation. Planning processes are involved in many school tasks. For instance, children works out the ways to learn to memorise words that given by teachers in spelling task. This activity facilitates a planful approach to learning and are at the same time encourages the children to learn to spell specific words.

Attention is a cognitive process that involves focus and concentration to stimulus when there are distractions. This functional unit concerns self directing, information selecting and persistence of responding. Naglieri and Das (1997c) describe attention as "a mental process by which the individual selectively focuses on particular stimuli while inhibiting responses to competing stimuli presented over time". This process stresses on the demand of the tasks that involve focused, selective, sustained and effortful activity. According to Naglieri (1999), focused attention refers to directed concentration toward a particular activity. While selective attention requires the inhibition of responses to distracting stimuli. Sustained attention refers to the variation of performance over time which can be influenced by the different amount of effort required. Example of attention task in academics is illustrated by the grammar task of selecting correct pronounce (he, his or him) in sentences such as "This bag belongs to __ and This is __ bag." This creates the environment with targets (the him) and the distracters (the he or the his) for the first sentence and vice versa.

Simultaneous is a cognitive process which integrates several different stimuli into a whole. In this process, individual have to acquire the ability of making connections between the pieces to be an overall concept. According to Naglieri and Das (1997c), "Simultaneous processing is a mental process by which the individual integrates separate stimuli into a single whole or group". The important key of this process is that the person must see how all the separate elements are interrelated in a conceptual whole. Simultaneous processing has strong spatial and logical dimensions for both nonverbal and verbal content. The spatial aspect refers to the perception of stimuli as a whole. In academic setting, simultaneous processing is involved in understanding grammatical statements that demand the integration of words into a whole idea. This integration involves comprehension of word relationships, prepositions and inflections so that the person can obtain meaning based on the whole idea (Naglieri 1999).

Successive is a cognitive process which applying existing information in more specific requirements. This process demands respondents to remember or use information that follows in a strict, defined order, especially serial and syntactical information. Naglieri and Das (1997c) describe successive processing as "a mental process by which the individual integrates stimuli into a specific serial order that forms a chain-like progression". The emphasis on the steps or successive processing is also involved in reading, especially in initial reading or 
decoding of unfamiliar words. This can be illustrated in the use of phonics in English language or suku kata in Malay language. Children must learn the association of the sounds, in correct order with the letters of the words. While in a sentence, children learn the order of words to form a grammatically correct sentence. For example "Who is this man?" and "This man is who?"

It is important to remember that all PASS processes are involved in most things people do. In reading as an example, planning is needed for organising how the task will be completed and for exerting impulse control. The ability to differentiate the letters or words requires heavy demand on attention. While simultaneous processing requires the individual to connect the meaning of each words in the sentences or the meaning of each sentence in the passage. Finally, successive process requires a person to have the ability to identify orderly words from a sentence or ideas of the sentence in the passage.

\section{RESEARCH INSTRUMENTS}

\subsection{Cognitive Assessment System (CAS)}

The cognitive functions of PASS were operationalised by Cognitive Assessment System (CAS), one of the latest technology based instrument in assessing the cognitive functions of brain. It was built in 1997 by Dr. Jack A. Naglieri and Dr. J. P. Das. According to Naglieri (1999), "the single most important goal of the CAS is to encourage an evolutionary step from the traditional Intelligence Quotient (IQ), general ability approach to a theory-based, multidimensional view with constructs built on contemporary research in human cognition". CAS consists of four subscales Planning, Attention, Simultaneous and Successive which are the process of Luria's three functional units of brain or the theory of PASS. The four subscales comprise three subtests for representing the whole score of the cognitive functions.

These are described below according to the PASS scale to which they belong. In Planning Scale, the first subtest Matching Numbers consists of four pages that contain eight rows of six numbers per row of each page. The subjects are instructed to underline the two numbers in each row that are the same. Numbers increase in length from one digit to seven digits across the four pages, with four rows for each digit length. Each item has a time limit. The subtest score is based on the combination of time and number of correct answers for each page. The second subtest, Planned Codes contains two pages, each with a distinct set of codes and arrangement of rows and columns. An example is shown at the top of each page how letters correspond to simple codes (e.g., A, B, C, and $\mathrm{D}$ correspond to $\mathrm{OX}, \mathrm{XX}, \mathrm{OO}$, and $\mathrm{XO}$, respectively). Every page contains seven rows and eight columns of letters without codes to be filled by subjects. The subjects are instructed to fill in the appropriate code in the empty box beneath each letter. On the first page, all the As appear in the first column, all the Bs in the second column, all the $\mathrm{Cs}$ in the third column, and so on. On the second page, letters are configured in a diagonal pattern. The subjects are allowed to complete each page in whatever fashion he or she wishes. The subtest score is based on the combination of time and number of correct answers for each page. The third subtest Planned Connections contains eight items. The first six items require the subjects to connect numbers appearing in a quasi-random order on a page in sequential order. The last two items require the subjects to connect both numbers and letters in sequential order, alternating between numbers and letters (e.g., 1-A-2-B-3-C). Items are constructed so that the subjects never complete a sequence by crossing one line over the other. The subtest score is based on the total amount of time in seconds used to complete the items.

The fourth subtest Attention Scale comprises subtest Expressive Attention which uses two different sets of items depending on the age of the subjects. Subjects who are eight years old and older are presented with three pages. On the first page, the subject reads colour words (i.e. BLUE, YELLOW, GREEN and RED) presented in quasi-random order. Next, the subjects name the colours of a series of rectangles (printed in blue, yellow, green and red). Finally, the words BLUE, YELLOW, GREEN and RED are printed in a different colour than the colours the words name. The subjects are instructed to name the colour ink of the words rather than to read the words of colours. The first two pages are to familiarise the subjects with the word and colour patterns. The score will be counted on the last page which is used as the measure of attention. The subtest score is based on the combination of time and number of correct answer. The second subtest Number Detection consists of pages of numbers that are printed in different formats. On each page, the subjects are required to find a particular stimulus (e.g. the numbers 1,2 , and 3 printed in an open font) on a page containing many distractors (e.g., the same numbers printed in a different font). There are 180 stimuli with 45 targets or $25 \%$ as targeted numbers on the pages. The subtest score reflects the ratio of accuracy (total number correct answer minus the number of false detections) to total time for each item summed across the items.

In Simultaneous Scale, Nonverbal Matrices, comprising a 33-item subtest, uses shapes and geometric designs that are interrelated through spatial or logical organization. The subjects are required to decode the relationships 
among the parts of the item and choose the best of six options to match a missing space in the grid. Every item is scored 1 as correct or 0 as incorrect. The subtest score is based on the total number of items correctly answered. Secondly, the Verbal-Spatial Relations subtest that consists of 27 items requires the comprehension of logical and grammatical descriptions of spatial relationships. The items contain six drawings and a printed question at the bottom of each page. Items involve both objects and shapes that are arranged in a specific spatial manner. For example, "Which picture shows an arrow pointing toward a circle that is in a square?" includes six drawings with various arrangements of geometric figures, only one of which matches the description. The examiner reads the question aloud, and the subjects are required to select the option that matches the verbal description. The subjects must indicate his or her answer within a 30 seconds time limit. The subtest score reflects the total number of items correctly answered within the time limit.

In Successive Scale, the first subtest Word Series requires the subjects to repeat words in the same order as stated by the examiner. The test consists of the following nine single-syllable and high-frequency words such as Book, Car, Cow, Dog, Girl, Key, Man, Shoe, Wall. The examiner reads 27 items to the subjects. Each series ranges in length from two to nine words. Words are presented at the rate of one word per second. Items are scored as correct if the subjects reproduce the entire word series. The subtest score is based on the total number of items correctly repeated. The second subtest Sentence Repetition requires the subjects to repeat 20 sentences that are read aloud. Each sentence is composed of colour words (e.g., "The purple yellow are green and the reds are white. Who are green?"). Words are presented at the rate of two words per second. The subjects are required to repeat each sentence exactly as presented. Colour words are used to reduce the influence demands of the syntax of the sentence in order to contain little semantic meaning. An item is scored as correct if the sentence is repeated exactly as presented. The subtest score reflects the total number of sentences repeated correctly. CAS reliability coefficient for standard full scale is 0.96 and basic full scale is 0.87 . The full average reliability coefficients for the four subscales are Planning (0.88), Attention (0.88), Simultaneous Processing (0.93), and Successive Processing (0.93).

\subsection{Wide Range Achievement Test -4 (WRAT-4)}

The second instrument used in this study was WRAT- 4. It consists of four subtests that measure the academic skills namely, Word Reading, Sentence Comprehension, Spelling, and Math Computation. In this study, there were three reading skills that involved representing the measure of reading processes such as decoding, comprehension and retention. It was standardized on a representative sample of over 3,000 individuals ranging from 5-94 years based on American norms. The Wide Range Achievement Test-Fourth Edition (WRAT- 4) is designed to provide "a quick, simple, psychometrically sound assessment of academic skills" (Wilkinson \& Robertson 2006). This study used the reading subtests of word reading, sentence comprehension and spelling that measured the three reading processes decoding, reading comprehension and retention as mentioned. Descriptions of the three reading subtests were stated as follow:

The first subtest of WRAT-4 is Word Reading that measures letter and word decoding through letter reading (15 items) and word reading (55 words). The letter reading section was administered to children who do not meet the basal scoring guidelines for the Word Reading subtest. It assumed to measure letter and word recognition rather than speech or dictation that representing the decoding process in reading.

Secondly, the Sentence Comprehension subtest measures an individual's ability to gain meaning from words and to comprehend ideas and information contained in sentences through the use of a modified cloze technique. The Sentence Comprehension subtest contains 50 items of one to two sentences each, where the participants fill in the blank with one or two words to indicate their comprehension of the sentence.

The third subtest of reading skills in WRAT-4 is Spelling subtest. It measures an individual's ability to encode sounds into written form through the use of a dictated spelling format containing both letters and words. The Spelling subtest is made up of two parts. The first part, administered to children aged 7 years or younger, includes letter writing (i.e., 13 letters must be written); the second part consists of 42 words that must be spelled correctly. Finally, Reading Composite is the score which combines the standard scores of the Word Reading and Sentence Comprehension subtests to provide a more comprehensive measure of reading achievement. According to Wilkinson \& Robertson (2006), the WRAT-4 standardization process includes samples of children with various disabilities, and subsequent studies were conducted to determine the capacity of the battery to discriminate among three groups children with learning disabilities, low cognitive skills, and high cognitive skills. This test is considered suitable to measure the poor ESL readers' reading ability level in Malaysian schools.

Although there are two alternative forms that can be used interchangeably in the WRAT- 4 which are the Blue Form and the Green Form. In this study, the only blue form was used for comparable results to avoid any doubt. 
The testing materials for administration of the WRAT-4 in this study includes blue response form for Spelling; test forms for recording the scores of the Word Reading and Spelling subtests; test forms for the Sentence Comprehension subtest; word reading list-spelling list cards; sentence comprehension cards and sentence comprehension sample cards. In addition, the research assistants were provided with needed stationeries such as pens, pencils and stopwatches. The research assistants were briefed and thoroughly trained to use the testing materials and procedures. For scoring, raw scores were obtained on each subtest and then converted to standard scores, based on a mean of 100 and a standard deviation of 15, according to age and grade normative groups as provided in the test manual. Wilkinson \& Robertson (2006) had conducted an analysis in reliability for the WRAT-4 to ensure the internal consistency and the coefficients overall that showed high levels of ranging from 0.92 to 0.98 . The test also has moderate levels of internal consistency within its subtests, with reliability coefficients ranging from 0.87 to 0.93 . The reading composite score coefficients are high to the ranging from 0.95 to 0.96 .

\subsection{Research Procedure}

This study starts to identify randomly standard three children at a national primary school. The subjects were among the children who learn English as the second language (ESL) in formal education. The samples involved have attended at least 2 years formal curriculum of English language. They have also exposed to the English language since attending the early education. However, none of the children spoke English as their first language at home and all of them were ESL children. This study had chosen standard three children as subjects study because this group of children had received a certain amount of English language input and expected to have adequate exposure in the ESL through out the first three years of formal primary education under the Malaysian education system. This academic level would be a crucial period for identifying children with learning difficulties especially in reading, mathematical calculation and writing $(3 M)$.

They were firstly assessed for the PASS cognitive processing by CAS and then reading component skills by WRAT-4. Cognitive Functions of PASS processes are Planning, Attention, Simultaneous and Successive; while reading processes are Word reading, Reading Comprehension and Spelling. It took about 45 minutes to administer CAS while about 30 minutes for WRAT-4. The basic battery of CAS was administered individually to assess cognitive processes and scores were obtained by rapid score software. In order to establish adequate rapport with each participant, the work was carried out in a separate room in the schools and maximum care was taken to avoid from any external disturbances during task administration. Some instructions and items of research instruments had been translated into Malay Language for better understanding of children in completing their tasks. CAS consists more of pictorial exposures, but some subtests of Simultaneous and Successive had been translated into Malay Language and its reliability and validity were tested. However, some minor but important determinants of understanding such as technical instructions and conceptual explanations were considered for both tests during administration. Standard scores $(M=100, S D=15)$ were obtained from the test manuals and used in all data analyses for both CAS and WRAT-4.

\section{DISCUSSION}

Table 1 shows Mean Scores of CAS Full Scale and each subscales. CAS profiles among the ESL learners are at average level of cognitive processing ability based on American standardization scores. The full scale of CAS mean scores was 98 which is the average level of cognitive processing among the subjects. While the WRAT-4 reading components show the mean score of overall reading performance $\mathrm{M}=90$ which was slightly at the average level. Correlation analysis revealed that cognitive processes were significantly correlated with overall reading performance with $r=0.789, p<0.01$. This explains that the level of cognitive processing is one of the significant determinants among the ESL learners in learning the language. It was not only the full scale score of CAS highly correlated with reading composite, specifically it was also significantly correlated with each of the reading components skills such as word reading $(r=0.759, p<0.01)$, reading comprehension $(r=0.714, p<0.01)$ and spelling $(r=0.780, p<0.01)$ among the ESL learners. (refer Table 1$)$

On the other hand, the correlational analysis of Table 2 revealed the cognitive processes of Planning and Simultanoeus had quite high and significant relationship with overall reading performance represented by reading composite which were $r=0.676, p<0.01$ and $r=0.724, p<0.01$ respectively. While Attention and Successive processing shows the moderate significant correlation with reading composite which were $r=0.550, p<0.01$ and $r=0.463, p<0.01$. The Simultaneous processing had strong relationship that was significantly correlated with reading comprehension $(r=0.710, p<0.01)$ and spelling $(r=0.730, p<0.01)$. These data suggested that the comprehension and spelling abilities among ESL learners tend to be strongly correlated with the Simulataneous processing following with Planning processing. However, the Attention and Successive processing were also 
related to the component skills of reading after the both processes. The Successive processing had low score of $r=$ $0.393, p<0.01$ for the correlation with reading comprehension. (refer Table 2)

This finding is supported by the study of Shamita, Das, Stack-Cutler and Parrila (2010) who investigated the pattern of relationships between two reading skills and the four cognitive processes. The study found that the relationship between word reading and reading comprehension significant $(r=0.81, p<0.001)$, but both skills were significantly related to simultaneous processing ( $r=0.62$ and $r=0.75, p<0.001$, respectively) as well as to the overall intellectual functioning (Full Scale) of the children $(r=0.44, p=0.02$ and $r=0.48, p=0.01)$. These results suggest that reading proficiency, as well as improvement in reading proficiency, is partly determined by one's proficiency in specific cognitive processes as reported in previous studies (e.g. Das et al. 1994). However, when both word reading and reading comprehension reach levels above the norm for the appropriate grade, as in the normal reading group, the two skills may become more independent of one another.

Similarities in the cognitive processes relevant to reading comprehension have been found for monolingual and ESL readers. Specifically, phonological processing, verbal working memory, and syntactic awareness can explain reading comprehension performance for native English speakers and ESL speakers (Low \& Siegel 2005). Vocabulary knowledge may play a key role in reading comprehension performance for ESL readers as well. Specifically, weak vocabulary knowledge of children learning a second language is likely to have an impact on their reading comprehension abilities (Hutchinson, Whiteley, Smith, \& Connors 2003; Sen \& Blatchford 2001). The cognitive processing of PASS would also be able to explain the language acquisitions ability besides reading. Kirby and Williams (1991) suggested that acquiring parts of speech demands processing elements of spoken language in a serial manner as well as perceiving words as a whole. This was supported by the study of Clay (1993) who emphasised the importance of not exclusively attending to the sounds of oral language but also grasping its sequential structure and detecting common sound patterns.

\section{CONCLUSIONS}

The purpose of this study was to explore the relationship of PASS cognitive processes and reading components skills among the ESL learners. At the same time, the study proposes which PASS cognitive processes best predict reading skills in language learning. CAS is one of the alternatives in providing such capabilities and in helping children with reading deficits. When intelligence is defined and measured as cognitive processes, it becomes relevant to performance on reading-related activities. Cognitive ability measures of the CAS have relevance to our understanding of reading. Teachers and educational psychologists should consider using CAS rather than the general or traditional intelligence tests in order to detect processes that are related to determine whether or not children demonstrate consistent or inconsistent patterns of processing information. Besides, CAS has detectable abilities in identifying defects of cognitive functions and it is correlated with reading difficulties and other corresponding learning difficulties among children at primary and secondary schools. Furthermore, research on cognitive approach to reading remediation by PASS processes will be essential in helping children with reading difficulties, especially for determining intervention at early primary level.

\section{References}

Clay, M. M. (1993). An observation survey of early literacy achievement. Auckland, N.Z. Portsmouth, NH: Heinemann.

Das, J.P., Naglieri, J.A. \& Kirby, J.R. (1994). Assessment of cognitive process. Needham Heights: Allyn \& Bacon.

Dirks, E., Spyer, G., Van Lieshout, E.C., \& De Sonneville, L. (2008). Prevalence of combined reading and arithmetic disabilities. Journal of Learning Disabilities, 41(5):460-73. http://dx.doi.org/10.1177/0022219408321128

Ellemen, A.M. (2009). Exploring dynamic assessment as a means of identifying children at-risk of developing comprehension difficulties. Dissertation of Dr. of Phil. in Special Education, Vanderbilt University. [Online] Available: http://etd.library.vanderbilt.edu/available/etd-07312009-015758/

Fein, E. C., \& Day, E. A. (2004). The PASS theory of intelligence and the acquisition of a complex skill: a criterion-related validation study of Cognitive Assessment System scores. Personality and Individual Differences, 37(6), 1123-1136. http://dx.doi.org/10.1016/j.paid.2003.11.017

Hutchinson, J. M., Whiteley, H. E., Smith, C. D. \& Connors, C. (2003). The developmental progression of comprehension-related skills in children learning EAL. Journal of Research in Reading, 26: 19-32. http://dx.doi.org/10.1111/1467-9817.261003 
Joseph, L. M., McCachran, M. E. \& Naglieri, J. A. (2003). PASS cognitive processes, phonological processes, and basic reading performance for a sample of referred primary-grade children. Journal of Research in Reading, 26: 304-314. http://dx.doi.org/10.1111/1467-9817.00206

Khaidzir bin Hj. Ismail \& Ooi Boon Keat. (2008). The Intelligence of Children with Reading Difficulties (RD) by Cognitive Assessment System (CAS). e-Bangi. Journal of Social Sciences and Humanities, 3(3):10. [Online] Available: http://pkukmweb.ukm.my/e-bangi/papers/2008/ooi08.pdf

Kirby, J. R. \& Williams, N. H. (1991). Learning problems: A cognitive approach. Toronto: Kagan and Woo.

Lesaux, N. K., Lipka, O. \& Siegel, L. S. (2006). Investigating cognitive and linguistic abilities that influence the reading comprehension skills of children from diverse linguistic backgrounds. Reading and Writing, 19: 99-131. http://dx.doi.org/10.1007/s11145-005-4713-6

Low, P. B., \& Siegel, L. S. (2005). A comparison of the cognitive processes underlying reading comprehension in native English and ESL speakers. Written Language and Literacy, 8(2), 207-231. http://dx.doi.org/10.1075/wll.8.2.09low

Luria, A. R. (1973). The working brain: An introduction to neuropsychology. New York: Basic Books.

Mishra, R. \& Stainthorp, R. (2007). The relationship between phonological awareness and word reading accuracy in Oriya and English: A study of Oriya-speaking fifth-graders. Journal of Research in Reading, 30: 23-37. http://dx.doi.org/10.1111/j.1467-9817.2006.00326.x

Naglieri, J.A. (1999). Essentials of CAS Assessment. New York: John Wiley \& Sons.

Naglieri, J.A. \& Das, J.P. (1988). Planning-Arousal-Simultaneous-Successive (PASS): A Model for Assessment. Journal of School Psychology, 26: 35-48. http://dx.doi.org/10.1016/0022-4405(88)90030-1

Naglieri, J.A. \& Das, J.P. (1997a). Cognitive Assessment System. Itasca: Riverside Publishing.

Naglieri, J.A. \& Das, J.P. (1997b). Cognitive Assessment System Administration and Scoring Manual. Itasca: Riverside Publishing.

Naglieri, J.A. \& Das, J.P. (1997c). Cognitive Assessment System Interpretive Handbook. Itasca: Riverside Publishing.

Naglieri, J. A., Delauder, B. Y., Goldstein, S. \& Schwebach, A. (2006). WISC-III and CAS: Which correlates higher with achievement for the clinical samples? School Psychology Quarterly, 21(1), 62-76. [Online] Available: http://psycnet.apa.org/journals/spq/21/1/62/

Naglieri, J. A., Rojahn, J. \& Matto, H. C. (2007). Hispanic and non-Hispanic children's performance on PASS cognitive processes and achievement. Intelligence, 35, 568-579. [Online] Available: http://www.iapsych.com/articles/naglieri2007.pdf

Sen, R. \& Blatchford, P. (2001). Reading in A language: Factors associated with progress in the young children. Educational Psychology, 2, 189-202. http://dx.doi.org/10.1080/01443410020043887

Shamita, Das, J.P., Stack-Cutler \& Parrila. (2010). Remediating Reading Comprehension Difficulties: A Cognitive Processing Approach. Reading Psychology, 31(5), 428-453. http://dx.doi.org/10.1080/02702710903054915

Shapiro, L.R., Hurry, J., Masterson, J., Wydell, T.N. \& Doctor, E. (2008). Classroom implications of recent research into literacy development: from predictors to assessment. Dyslexia, 15(1), 1-22. http://dx.doi.org/10.1002/dys.380

Siegel, L.S. \& Lipka, O. (2007). The development of reading skills in children with English as a second language. Scientific Studies of Reading, 11, 105-131. http://dx.doi.org/10.1080/10888430709336555

Wilkinson, G.S. \& Robertson, G.J. (2006). Wide Range Achievement Test 9 WRAT-4: Professional Manual. Psychological Assessment Resources (PAR), Inc. 
Table 1. Mean Scores of CAS Full Scale and each subscales

\begin{tabular}{llcc}
\hline Test & Subscales & M & SD \\
\hline CAS & Planning & 105.27 & 14.02 \\
& Simultaneous & 89.92 & 15.31 \\
& Attention & 102.18 & 12.17 \\
& Successive & 97.77 & 13.33 \\
& Full Scale & 98.13 & 14.49 \\
& & & \\
WRAT-4 & Word Reading & 103.54 & 23.01 \\
& Comprehension & 78.27 & 14.29 \\
& Spelling & 89.10 & 24.12 \\
& Reading Composite & 89.82 & 18.63 \\
\hline
\end{tabular}

$\mathrm{M}=$ Mean; $\mathrm{SD}=$ Standard Deviation; $\mathrm{N}=100$

Table 2. Inter-correlation of PASS and WRAT-4 Reading Subtests

\begin{tabular}{|c|c|c|c|c|c|c|c|c|}
\hline & Comprehension & Spelling & $\begin{array}{c}\text { Reading } \\
\text { Composite }\end{array}$ & Planning & Simultaneous & Attention & Successive & $\begin{array}{l}\text { Full } \\
\text { Scale }\end{array}$ \\
\hline Word Reading & $.751 * *$ & $.889 * *$ & $.963 * *$ & $.649 * *$ & $.660 * *$ & $.552 * *$ & $.461 * *$ & $.759 * *$ \\
\hline Comprehension & & $.827 * *$ & $.901 * *$ & $.626 * *$ & $.710 * *$ & $.453 * *$ & $.393 * *$ & $.714 * *$ \\
\hline Spelling & & & $.921 * *$ & $.655 * *$ & $.730 * *$ & $.592 * *$ & $.415 * *$ & $.780 * *$ \\
\hline Reading Composite & & & & $.676 * *$ & $.724 * *$ & $.550 * *$ & $.463^{* *}$ & $.789 * *$ \\
\hline Planning & & & & & $.530 * *$ & $.644 * *$ & $.367 * *$ & $.823 * *$ \\
\hline Attention & & & & & & $.559 * *$ & $.324 * *$ & $.795 * *$ \\
\hline Simultaneous & & & & & & & $.293 * *$ & $.798 * *$ \\
\hline Successive & & & & & & & & $.654 * *$ \\
\hline
\end{tabular}

$* * \mathrm{P}<0.01$ 